\title{
Relating near-Earth observations of an interplanetary coronal mass ejection to the conditions at its site of origin in the solar corona
}

\author{
A. N. Fazakerley, ${ }^{1}$ L. K. Harra, ${ }^{1}$ J. L. Culhane, ${ }^{1}$ L. van Driel-Gesztelyi, ${ }^{1,2,3}$ E. Lucek, ${ }^{4}$ \\ S. A. Matthews, ${ }^{1}$ C. J. Owen, ${ }^{1}$ C. Mazelle, ${ }^{5}$ A. Balogh, ${ }^{4}$ and H. Rème ${ }^{5}$ \\ Received 4 March 2005; revised 28 April 2005; accepted 25 May 2005; published 8 July 2005.
}

[1] A halo coronal mass ejection (CME) was detected on January 20, 2004. We use solar remote sensing data (SOHO, Culgoora) and near-Earth in situ data (Cluster) to identify the CME source event and show that it was a long duration flare in which a magnetic flux rope was ejected, carrying overlying coronal arcade material along with it. We demonstrate that signatures of both the arcade material and the flux rope material are clearly identifiable in the Cluster and ACE data, indicating that the magnetic field orientations changed little as the material traveled to the Earth, and that the methods we used to infer coronal magnetic field configurations are effective. Citation: Fazakerley, A. N., L. K. Harra, J. L. Culhane, L. van Driel-Gesztelyi, E. Lucek, S. A. Matthews, C. J. Owen, C. Mazelle, A. Balogh, and H. Rème (2005), Relating near-Earth observations of an interplanetary coronal mass ejection to the conditions at its site of origin in the solar corona, Geophys. Res. Lett., 32, L13105, doi:10.1029/ 2005 GL022842.

\section{Introduction}

[2] ICMEs which engulf the Earth's magnetosphere are often associated with magnetospheric storms, typically when they exhibit fast flow $\left(>500 \mathrm{kms}^{-1}\right)$ and strong southward magnetic field components $\left(\mathrm{GSE} \mathrm{B}_{\mathrm{Z}}<0\right)$. Successful storm prediction will require (at least) the ability to reliably identify an Earth-directed CME as it leaves the Sun, to determine its speed and to infer whether the ICME will exhibit $\mathrm{B}_{\mathrm{Z}}<0$. Evolution of the magnetic field during transit is predicted [e.g., Cargill and Schmidt, 2002] but observational studies are required to quantify this evolution and its consequences. Previous studies have tried to relate solar source region characteristics to those of the interplanetary ejecta. Bothmer and Rust [1997] found that magnetic clouds (MCs) associated with filament disappearances were generally left-handed for Northern hemisphere filaments and vice versa for the Southern hemisphere. Leamon et al. [2002] made a statistical study of the relationship between S and inverse S-shaped (sigmoidal) regions in the corona and associated MCs, and found that neither a sigmoidal flux rope model nor a large-scale dipole model reliably predicted

\footnotetext{
${ }^{1}$ Mullard Space Science Laboratory, University College London, Dorking, UK

${ }^{2}$ Also at Konkoly Observatory, Hungarian Academy of Sciences, Budapest, Hungary.

${ }^{3}$ Also at Observatoire de Paris, Meudon, France.

${ }^{4}$ Space and Atmospheric Physics Group, Imperial College of Science, Technology, and Medicine, London, UK.

${ }^{5}$ Centre d'Etude Spatiale des Rayonnements, Toulouse, France.
}

Copyright 2005 by the American Geophysical Union. 0094-8276/05/2005GL022842 the MC flux rope. Leamon et al. [2004] proposed that a better model would invoke reconnection between active regions and their large scale overlying fields during the eruption [see also Antiochos et al., 1999]. In contrast, Mandrini et al. [2005] have studied a case involving a very small source region and shown that the orientation and helicity of their MC matched that of the pre-eruption minisigmoid. Webb et al. [2000] used modeling to successfully identify the origins of a halo CME observed in May 1997. McAllister et al. [2001] successfully predicted the topology of a $\mathrm{MC}$ based on observations of the overlying arcade and surrounding magnetic structure. Here, we examine an ICME that originates in a non-sigmoidal active region. The magnetic configuration observed near the Earth is well matched to the pre-ejection coronal configuration, in contrast to some of the earlier results outlined above.

\section{Near-Earth Observations: January 22, 2004}

[3] At $\sim$ 01:35 UT on January 22, 2004, the Cluster spacecraft, located at $(15.0,-11.1,5.2) \mathrm{R}_{\mathrm{E}} \mathrm{GSE}$ in the solar wind, measured a strong interplanetary shock, followed by a 7 hour interval of compressed solar wind plasma (referred to below as "sheath"), and then by the leading edges of the coronal ejecta (Figure 1a). After a further $\sim 2$ hours, the ejecta magnetic field turned southward and remained so for 24 hours, while the flow speed remained above $500 \mathrm{kms}^{-1}$. Such conditions favour the occurrence of a geomagnetic storm, and a storm was indeed observed with provisional minimum Dst of $-149 \mathrm{nT}$ at 14:00 UT.

[4] The ACE spacecraft, located at (229.8, -40.6, 22.1) $\mathrm{R}_{\mathrm{E}}$ GSE detected the same phenomena $\sim 37$ minutes earlier. Cluster later moved downstream of the terrestrial bowshock and so did not provide unbroken coverage, but ACE recorded the entire event (Figure 1b). No shocks were seen at ACE during the preceding 6 days.

[5] The shock characteristics were examined using the 4 Cluster spacecraft, which were in a tetrahedral configuration, roughly $200 \mathrm{~km}$ apart. The FGM magnetometer data time resolution (22 vectors $\mathrm{s}^{-1}$ ) was sufficient to clearly identify the differing shock arrival times at the four spacecraft. Assuming the shock front was locally planar, a simple 4-spacecraft timing analysis provides an estimate of the shock normal vector and velocity. This method is free of the difficulties associated with single spacecraft methods [Schwartz, 1998]. The sunward shock normal direction was $n=(0.91,-0.31,-0.29)$ GSE which is tilted at a cone angle of $25^{\circ}$ to the Sun-Earth line. The (anti-sunward) shock speed along this normal is $\mathrm{V}_{\mathrm{E}}=740 \mathrm{~km} / \mathrm{s}$. The shock is quasi-perpendicular $\left(\theta_{\mathrm{BN}}=80^{\circ}\right)$ and supercritical with an Alfvén Mach number of 5.6. 


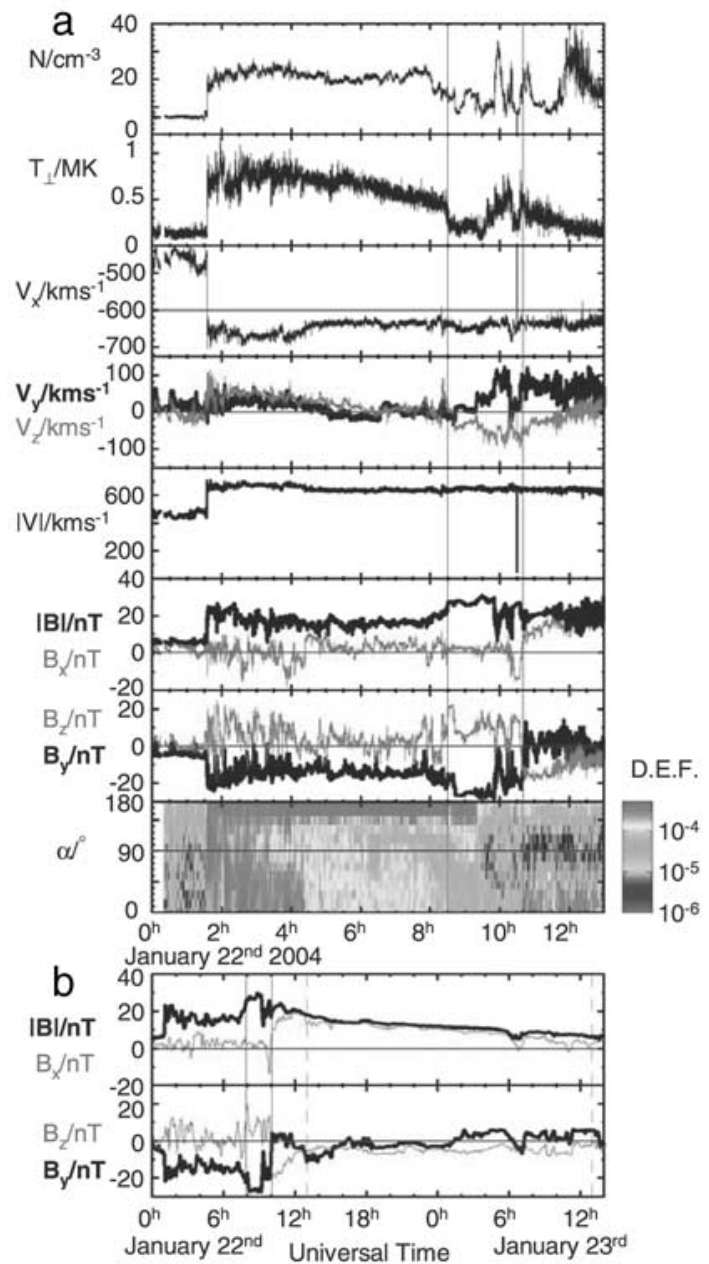

Figure 1. (a) Cluster data: CIS-HIA ion number density, perpendicular temperature, and bulk flow velocity; FGM magnetic field vector; PEACE suprathermal $(\sim 130 \mathrm{eV})$ electron differential energy flux vs. pitch angle $\left[\mathrm{ergs} /\left(\mathrm{cm}^{2} \mathrm{~s}\right.\right.$ $\mathrm{sr} \mathrm{eV})$ ]. (b) ACE MAG magnetic field vector. All vectors in GSE; thicker line for y component or $|\mathrm{B}|$ in relevant panels. See color version of this figure in the HTML.

[6] The sheath plasma exhibited the same composition as the solar wind, but density, temperature and magnetic field strength were higher than solar wind levels. PEACE electron spectrometer data shows unidirectional suprathermal electrons with pitch angle $\alpha=180^{\circ}$ in the solar wind and sheath, consistent with electrons streaming away from the Sun on open magnetic field lines. The sheath material flowed predominantly anti-sunward, but the flow was inclined at $\sim 5^{\circ}$ (reducing over time) to the solar wind, tilted towards $+z$ and to a lesser extent $+y$ GSE. The shock motion and sheath flows suggest that the main body of the ICME passed by the Earth some distance away in the $[-y,-z]$ GSE direction.

[7] The last sheath material passed Cluster at $\sim 08: 30$ UT, followed by plasma of coronal origin, as indicated by $\mathrm{He}^{2+} / \mathrm{H}^{+}$ratios which increased at this time and remained elevated over solar wind levels for a further 28 hours (not shown). Between $\sim 08: 30$ and $\sim 10: 40$ UT, the magnetic field lay in the $y-z$ plane, largely along $-y$ GSE but with a small $+z$ component, and its magnitude $|B|$ was elevated above sheath levels (Figure 1, between solid vertical lines). Suprathermal electrons were observed with $\alpha=180^{\circ}$, suggesting that these are open field lines. Within this interval, between 09:50 and 10:20 UT, was a region of depressed $|B|$, associated with two distinct regions of higher density and temperature plasma, coinciding with suprathermal electrons with $60^{\circ}<\alpha<120^{\circ}$, perhaps locally pitch angle scattered in this region of higher plasma beta as proposed by Crooker et al. [2003]. During the corresponding interval observed upstream at ACE, SWICS data show $\mathrm{O}^{7+} / \mathrm{O}^{6+}$ ratios a factor 20 above solar wind levels. These observations are consistent with the spacecraft having sampled a region of trapped coronal plasma. At the end of this period, the magnetic field swings a little to the anti-sunward, and strong fluxes of $120^{\circ}<\alpha<180^{\circ}$ electrons (and also $>10 \mathrm{keV}$ protons) appear at Cluster, which we interpret as foreshock particles from the terrestrial bowshock.

[8] Between 10:36 and 10:48 UT the magnetic field rotated to a predominantly $[+x,-z]$ GSE direction. A minimum variance analysis (with well separated eigenvalues, $\lambda_{1}=283, \lambda_{2}=28.1, \lambda_{3}=1.7$ ) indicated a tangential discontinuity, in which the magnetic field rotates through $180^{\circ}$ in a plane with the normal $(0.83,-0.22,0.51)$. We interpret this as a current sheet separating two distinct plasmas of coronal origin. Note that the plane is tilted northwards at $\sim 30^{\circ}$ to the GSE $y-z$ plane, which is not consistent with lying on a simple spherical expansion front. Cluster (foreshock electrons notwithstanding) and ACE data (not shown) confirm that a bi-directional suprathermal electron signature began at this point and persisted thereafter throughout the interval discussed in this paper.

[9] Cluster re-entered the terrestrial magnetosheath at 16:30 UT, however ACE data suggest that the predominantly sun-pointing ICME magnetic field configuration persisted for about a day, during which time the flow speed dropped, the density declined and the magnetic field weakened, as is typical for an expanding ICME. Minimum variance analysis from January 22, 13:00 UT to January 23, 13:00 UT (Figure $1 \mathrm{~b}$ between dashed vertical lines) shows a $\sim 180^{\circ}$ rotation in a plane with normal along the minimum eigenvector $(+0.11,+0.17,-0.98)$. The magnetic field vector shows a steady $B_{Z}<0$, a $B_{X}>0$ that gradually weakens, and a $\mathrm{B}_{\mathrm{y}}$ that fairly steadily transforms from $<0$ to $>0$. We interpret this as a crossing of a flux rope, in which the core field is directed North to South (N-S) and is surrounded by a magnetic field with a left-handed twist. The spacecraft passes to the West (-ve y GSE) side of the axis. As there is no magnetic field peak during the crossing, the spacecraft may be far from a core axial field if one exists.

[10] Preceding this interval, between 10:40 and 13:00 UT, $|B|$ appears enhanced relative to the trend of the next 24 hours, $\mathrm{B}_{\mathrm{y}} \sim 0, \mathrm{~B}_{\mathrm{x}}>0$ and $\mathrm{B}_{\mathrm{z}}$ is strongly negative. Bi-directional electrons are seen here, so we believe that this interval belongs to the flux rope, although the magnetic field direction is unexpected. We tentatively attribute its differing character to the effects of compression and possibly other interactions (e.g., reconnection) as the flux rope pushes against the upstream plasma.

\section{Solar Observations: 19-20 January 2004}

[11] AR0540, located at S14W36, on January 20, was a magnetically complex region which produced several large 


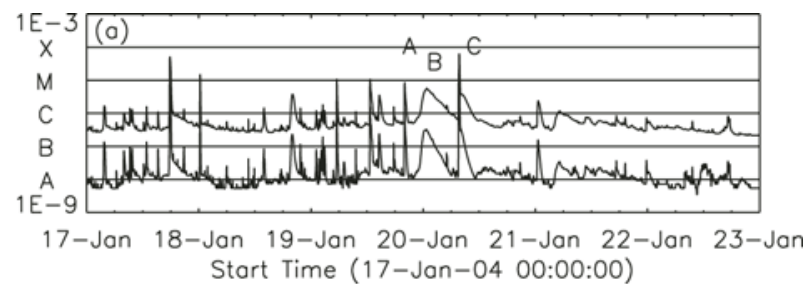
Start Time (17-Jan-04 00:00:00)

(b)

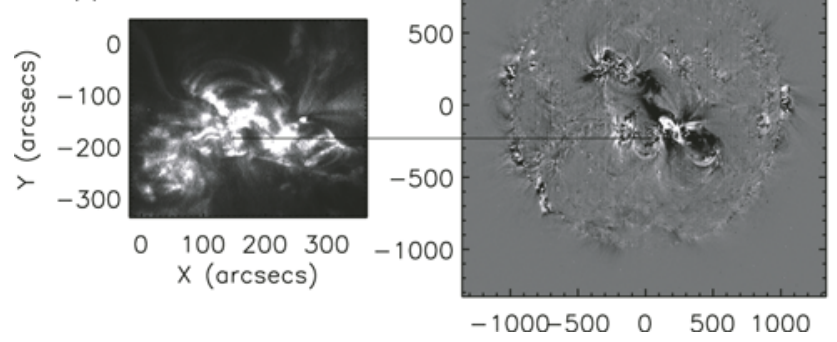

Figure 2. (a) GOES light curve showing flaring activity. Labels A, B and C indicate events that may be associated with CME impacts at Earth. (b) EIT $195 \AA$ image showing flare B location in AR 0540. (c) EIT difference image showing CME-related coronal dimming regions NE and SW of (white) active region.

flares (Figure 2a). Activity then dropped with the decay of the region. There were 3 significant flares in the period January 19, 20:00 UT to January 20, 10:00 UT that could be related to the event seen at Earth (see Figure 2 and Table 1); a C-class impulsive flare (A), followed by a long duration C-class flare that lasted more than 6 hours (B) and finally a complex three stage M-class flare (C).

[12] In order to determine which flare was related to the ICME, we sought to estimate travel times of the ICME and/ or the associated shock. Flare A had an associated metric Type-II burst observed at Culgoora with an estimated shock speed of $\sim 450 \mathrm{kms}^{-1}$ (treating the data as indicative of an outward propagating shock in the corona). An unrelated non-halo CME occurred slightly before Flare A. Flare B had a related halo CME, but no metric type II emission. There is a weak indication of a shock in WIND/Waves data (Type II burst) (M. L. Kaiser, private communication). Flare C had three distinct phases, all of which had quadrupolar magnetic configurations. We associate Flares $\mathrm{Ci}$ and $\mathrm{Cii}$ with two metric Type II radio bursts observed at Culgoora, with corresponding estimated shock speeds of $\sim 550 \mathrm{kms}^{-1}$ and $\sim 850 \mathrm{kms}^{-1}$. A non-halo CME was detected by SOHO-LASCO-C2 at 08:30 UT.

[13] In the absence of a well-defined near-Sun shock signature for Flare B, we applied the cone model of Michalek et al. [2003] to the halo CME. We estimated that the angle between the CME cone symmetry axis and the sky plane was $\gamma \sim 60^{\circ}$, the cone opening angle was $\alpha \sim 150^{\circ}$ and the CME velocity $\mathrm{V}_{\mathrm{B}} \sim 910 \mathrm{kms}^{-1}$. The cone symmetry axis lies at $\sim 20^{\circ}$ to solar N-S such that the first detection in LASCO C2 occurred in the solar SW quadrant.

[14] Table 1 summarizes the details given above, and for each flare provides two estimates of the shock arrival time at Earth; $T_{1}$ for travel at the estimated near-Sun speed or $T_{2}$ for travel at the speed measured by Cluster near Earth. We conclude that shocks from Flares $\mathrm{A}$ and $\mathrm{C}$ must arrive too late, while a shock from Flare B can arrive at Cluster at the observed time. We conclude that the source of the shock observed at Earth was the halo CME associated with the long-duration flare that began at 23:08 UT on January 19, in AR 0540 .

[15] Long duration flares are assumed to occur following the reconnection of sheared arcade fields beneath a rising CME. They often, but not always, involve a filament eruption. In this case no filament eruption was observed. The diverging flare ribbons and the persistent formation of flare loops connecting them (i.e. reforming arcades, Figure $2 \mathrm{~b}$ ) support the reconnection picture. EIT difference images, in which two extended dimming regions appeared $\mathrm{NE}$ and SW of the active region (Figure 2c) may be interpreted as the footprints of magnetic field lines which expanded in the CME. In this event, reconnection of sheared arcade fields can be expected to generate a flux rope with many turns, as observed by Cluster and ACE, whether or not a flux rope was present in the pre-CME configuration [e.g., Forbes and Isenberg, 1991; Antiochos et al., 1999]. The chirality or orientation of a pre-existing flux rope is unaffected in this scenario. MDI magnetogram data were used to identify the magnetic inversion line. Inspection of the magnetic polarity either side of it ( $>0$ to the SE, $<0$ to the NW) and the orientation of the arcade loops relative to the line, shows that the axial field of the flux rope was $\sim$ NNE-SSW oriented [Martin and McAllister, 1997]. The shear angle between the arcade loops and the inversion line suggests that the active region, and thus also the flux rope, has a left handed twist, somewhat unexpectedly for a southern hemisphere case [Pevtsov and Balasubramaniam, 2003]. The erupting flux rope is expected to carry away material associated with the overlying ESE-WNW oriented (i.e. potential, current-free) arcade field lines.

[16] LASCO images show an interaction between the CME and a streamer structure to the East of it (see Figure 3). Inspection of MDI magnetograms and time sequences of EIT images demonstrate that the open streamer magnetic field lines have the opposite polarity to the neighbouring arcade field lines. As the overlying arcade was forced to expand during the flux tube eruption, it is possible that the arcade magnetic field could have reconnected with the streamer magnetic field.

\section{Relating Solar and Near-Earth Observations}

[17] The cone model of the CME implies a central axis directed along $[-x,-y,-z]$ GSE, and a hemispherical shock front. The Cluster determination of a shock surface moving anti-sunward, towards $[+y,+z]$ GSE and measured sheath flows in the same sense are consistent with such a picture, and thus support the model.

Table 1. Event Timings

\begin{tabular}{lccccc}
\hline UT & Flare & Type II & CME & $\mathrm{T}_{1}$ & $\mathrm{~T}_{2}$ \\
\hline A & $19: 54$ & $20: 05-25$ & $19: 32$ & 23rd 15:55 & 22nd 03:54 \\
B & $23: 08$ & none & $00: 06$ & 21st 21:15 & 22nd 07:54 \\
Ci & $7: 30$ & $07: 39-50$ & n/a & 23rd 10:42 & 22nd 15:24 \\
Cii & $7: 40$ & $07: 48-57$ & $08: 30$ & 22nd 08:22 & 22nd 15:36 \\
Ciii & $8: 00$ & n/a & n/a & n/a & 22nd 15:54 \\
\hline
\end{tabular}




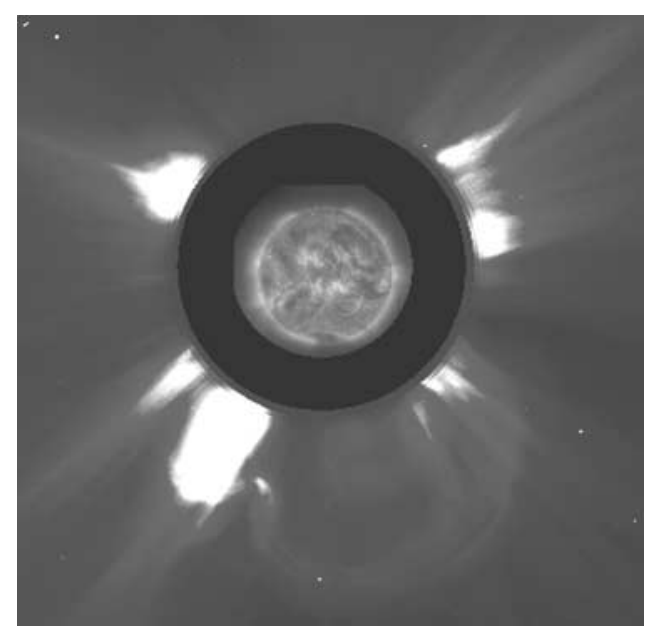

Figure 3. LASCO C2 coronagraph image at January 20, 00:54 UT, showing the halo CME. Note its interaction with the streamer (bottom left). See color version of this figure in the HTML.

[18] In the interplanetary ejecta, we expect to see first the appearance of an ESE-WNW and later a twisted NNE to SSW oriented magnetic structure.

[19] Cluster and ACE data show that the ICME material during the first 2 hours after the sheath passes has a coronal composition, and a magnetic field which was stronger than in the sheath, oriented primarily along $-y$ GSE with a distinct $+z$ GSE component, i.e. solar ESE-WNW. We identify this as magnetic flux from overlying arcade material, and suggest that the strong magnetic field is due to compression between the expanding flux rope and the upstream solar wind/sheath. The denser, hotter $(0.5 \mathrm{MK})$ ions, and high $\mathrm{O}^{7+} / \mathrm{O}^{6+}$ ratios indicative of higher altitude coronal plasma seen during this period may be the signatures of overlying arcade plasma that has been carried along with the ICME. The observation of anti-parallel-only rather than bi-directional electron fluxes during this period can be understood if the overlying arcade field lines did reconnect with streamer field lines to the East of the arcade, creating open field lines rooted in the corona only in the West.

[20] We interpret the 24 hour period (starting January 22, 13:00 UT) of ACE data with consistent IMF $\mathrm{B}_{\mathrm{Z}}<0$ and $\mathrm{B}_{\mathrm{X}}>0$ as the spacecraft passing through the flux rope which emerged with the CME. We infer that the spacecraft passed to the west of the rope axis, as expected if the main body of the rope maintained its coronal $\sim \mathrm{NNE}-\mathrm{SSW}$ orientation. The flux tube axis determined using ACE data lies N-S, i.e. slightly inclined relative to the coronal orientation, suggesting a local distortion of the flux rope, or else reflecting error margins in our analysis. The difficulties of fully characterizing an ICME flux rope using single spacecraft data are highlighted by Riley et al. [2004].

\section{Conclusion}

[21] We report a study in which we unambiguously identify the coronal source region and flare event that produced a halo CME which, now as an ICME, was detected in situ by Cluster and ACE. The source event was a long duration flare, which is expected to be related to the eruption of a magnetic flux rope, carrying with it overlying coronal arcade plasma. The expectation is confirmed by magnetic field and plasma signatures in the ICME which closely match their coronal counterparts, apparently having changed little while traveling from Sun to Earth.

[22] Acknowledgments. We thank the TRACE, SOHO, ACE and Cluster teams as well as the MSSL Solar UK Research Facility (SURF) and the ACE Science Centre. SOHO and cluster are joint projects of ESA and NASA. We also thank P. Démoulin, M. Kaiser and H. Kurokawa for their helpful support. LvDG acknowledges the Hungarian Government Grant OTKA T-039013. We thank the referees for their careful work.

\section{References}

Antiochos, S. K., C. R. DeVore, and J. A. Klimchuk (1999), A model for coronal mass ejections, Astrophys. J., 510, 485-493.

Bothmer, V., and D. M. Rust (1997), The field configuration of magnetic clouds and the solar cycle dependence, in Coronal Mass Ejections, Geophys. Monogr. Ser, vol 99, edited by N. Crooker, J. A. Joselyn, and J. Feynmann, p. 139-146, AGU, Washington D. C.

Cargill, P. J., and J. M. Schmidt (2002), Modelling interplanetary CMEs using magnetohydrodynamic simulations, Ann. Geophys., 20, 879-890.

Crooker, N. U., D. E. Larson, S. W. Kahler, S. M. Lamassa, and H. E. Spence (2003), Suprathermal electron isotropy in high-beta solar wind and its role in heat flux dropouts, Geophys. Res. Lett., 30(12), 1619, doi:10.1029/2003GL017036.

Forbes, T. G., and P. A. Isenberg (1991), A catastrophe mechanism for coronal mass ejections, Astrophys. J., 373, 294-307.

Leamon, R. J., R. C. Canfield, and A. A. Pevtsov (2002), Properties of magnetic clouds and geomagnetic storms associated with eruption of coronal sigmoids, J. Geophys. Res., 107(A9), 1234, doi:10.1029/ 2001JA000313.

Leamon, R. J., R. C. Canfield, and A. A. Pevtsov (2004), Helicity of magnetic clouds and their associated active regions, J. Geophys. Res., 109, A05106, doi:10.1029/2003JA010324.

Mandrini, C. H., S. Pohjolainen, S. Dasso, L. M. Green, P. Démoulin, L. van Driel-Gesztelyi, C. Copperwheat, and C. Foley (2005), Interplanetary flux rope ejected from an X-ray bright point. The smallest magnetic cloud source region ever observed, Astron. Astrophys., 434(2), $725-740$.

Martin, S. F., and A. H. McAllister (1997), Predicting the sign of helicity in erupting filaments and coronal mass ejections, in Coronal Mass Ejections, Geophys. Monogr. Ser., vol. 99, edited by N. U. Crooker, J. A. Joselyn, and J. Feynman, pp. 127-138, AGU, Washington, D. C.

McAllister, A. H., S. F. Martin, N. U. Crooker, R. P. Lepping, and R. J. Fitzenreiter (2001), A test of real-time prediction of magnetic cloud topology and geomagnetic storm occurrence from solar signatures, J. Geophys. Res., 106, 29,185-29,194.

Michalek, G., N. Gopalswamy, and S. Yashiro (2003), A new method for estimating widths, velocities, and source location of halo coronal mass ejections, Astrophys. J., 584, 472-478.

Pevtsov, A. A., and K. S. Balasubramaniam (2003), Helicity patterns on the Sun, Adv. Space Res., 32, 1867-1874.

Riley, P., et al. (2004), Fitting flux ropes to a global MHD solution: A comparison of techniques, J. Atmos. Sol. Terr. Phys., 66, 1321-1331.

Schwartz, S. J. (1998), Shock and discontinuity normals, Mach numbers and related parameters, in Analysis Methods for Multi-Spacecraft Data, ISSI Sci. Rep. SR-001, edited by G. Paschmann and P. W. Daly, pp. 249270, Int. Space Sci. Inst., Bern.

Webb, D. F., R. P. Lepping, L. F. Burlaga, C. E. DeForest, D. E. Larson, S. F. Martin, S. P. Plunkett, and D. M. Rust (2000), The origin and development of the May 1997 magnetic cloud, J. Geophys. Res., 105, $27,251-27,259$

A. Balogh and E. Lucek, Space and Atmospheric Physics Group, Imperial College of Science, Technology and Medicine, London SW7 2BZ, UK

J. L. Culhane, A. N. Fazakerley, L. K. Harra, S. A. Matthews, C. J. Owen, and L. van Driel-Gesztelyi, Mullard Space Science Laboratory, University College London, Holmbury St. Mary, Dorking RH5 6NT, U.K. (anf@mssl.ucl.ac.uk)

C. Mazelle and H. Rème, Centre d'Etude Spatiale des Rayonnements, 9 Ave. du Colonel Roche, B.P. 4346, F-31028 Toulouse Cedex 4, France. 